\section{Non-Invasive Flow Ratio (NiFR) Measurement based on Angiography Images}

\author{
Mohammad Sadeghian ${ }^{1}$, Vahid Mohammadi', Akbar \\ Shafiee ${ }^{2}$, Hamidreza Babakhani3*๑
}

\begin{abstract}
Background: Fractional flow reserve (FFR) is a gold standard to assess the impact of stenosis on the blood flow. The FFR method enhances diagnostic accuracy, lessens the need for stenting, and reduces costs. However, FFR is used in less than $10 \%$ of percutaneous coronary intervention (PCI) procedures because it needs pressure wires to measure the distal and proximal pressures and adenosine to create hyperemic conditions. Pressure-wire-based FFR measurement is, therefore, expensive and invasive.

Objective: This study aims to introduce a new approach on the basis of 3D coronary angiography and the Thrombolysis in Myocardial Infarction (TIMI) frame count for fast computation of FFR in patients with coronary artery disease.

Material and Methods: In this simulation study, we herein introduce Non-Invasive Flow Ratio drawing upon CFD to measure FFR based on coronary angiography images with less run time. In this study, 3D geometry was created based on coronary angiography images. The mean volumetric flow rate was calculated using the TIMI frame count. FFR calculated based on CFD was compared with pressure-wire-based FFR and NiFR was calculated in 85 patients.
\end{abstract}

Results: The NiFR $(r=0.738, P<0.001)$ exhibited a strong correlation with pressure-wire-based FFR. The result indicated that FFR was higher than 0.8 in the arteries with non-significant stenosis and lower than 0.8 in the arteries with significant stenosis.

Conclusion: The computational simulation of FFR and hemodynamic parameters such as pressure drop is a safe, efficient, and cost-effective method to evaluate the severity of coronary stenosis.

Citation: Sadeghian M, Mohammadi V, Shafiee A, Babakhani H. Non-Invasive Flow Ratio (NiFR) Measurement based on Angiography Images. J Biomed Phys Eng. 2021;11(6):685-692. doi: 10.31661/jbpe.v0i0.1160.

\section{Keywords}

Fractional Flow Reserve, Myocardial; Coronary angiography; Coronary Artery Disease

\section{Introduction}

T he aggregation of lipid deposits leads to stenosis and flow disturbance in arteries. This situation is termed "atherosclerosis", which results in a large number of diseases in the circulation system. The occurrence of this condition is frequent in coronary arteries and leads to occlusion and stroke. Currently, stent placement and coronary artery bypass graft surgery are effective methods for the treatment of atherosclerosis. Pressure-wire-based fractional flow reserve (FFR) is used to assess the severity of coronary stenosis and plan the revascu-
${ }^{1} \mathrm{MD}$, Department of Cardiology, Imam Khomeini Hospital, Tehran University of Medical Sciences,

Tehran, Iran

${ }^{2} \mathrm{MD}$, Department of

Cardiovascular Re-

search, Tehran Heart

Center, Tehran University

of Medical Sciences,

Tehran, Iran

${ }^{3} \mathrm{MSc}$, Department of

Mechanical Engineering,

Tarbiat Modarres Univer-

sity, Tehran, Iran

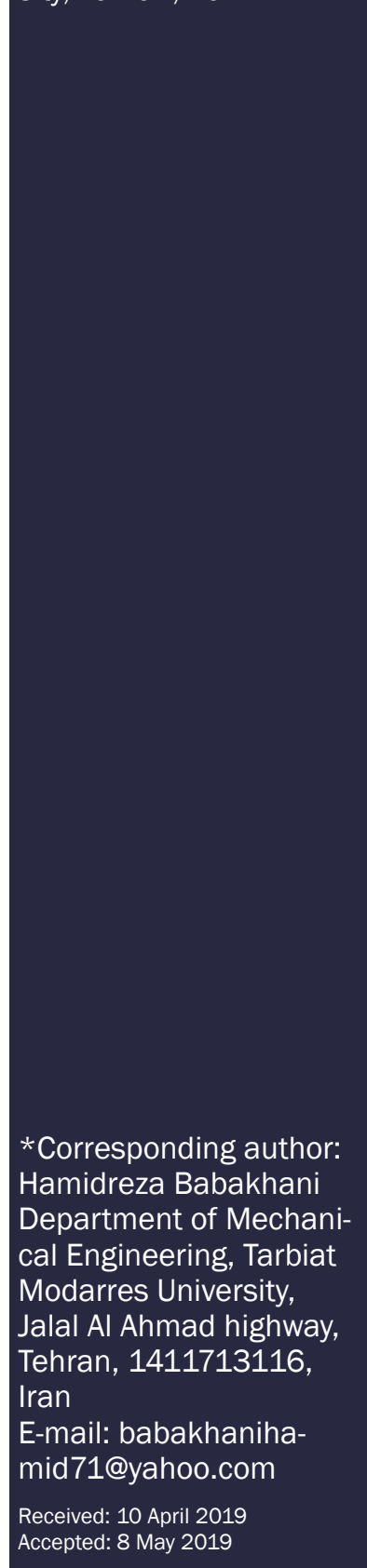


larization procedure. FFR is, therefore, a useful tool in angiography. The FFR method is considered as the gold standard to evaluate the significance of lesions with intermediate and borderline stenosis [1]. The FFR-guided procedure can confer favorable clinical outcomes inasmuch as it can boost diagnostic accuracy, lessen the need for stenting, and reduce costs. Nonetheless, FFR is measured in less than $10 \%$ of percutaneous coronary intervention (PCI) procedures because it needs pressure wires to measure the distal and proximal pressures and adenosine to create hyperemic conditions. FFR measurement is, therefore, expensive and invasive. New research shows that the computational simulation of blood pressure and flow based on computer equations can help diagnose and treat arterial occlusive disease [2].

A sudden reduction in the cross-section of an artery causes a pressure drop in the near stenosis region. According to the constraints on the determination of FFR, the simulation of computational fluid dynamics (CFD) is currently considered as a method overcoming these problems as it can calculate FFR without the need for pressure wires and adenosine [3].

$\mathrm{Tu}$ et al. [4] investigated pressure drop and velocity vectors in pre- and post-stenosis regions with the numerical simulation of the geometry of the diagonal artery and the left anterior descending artery (LAD) derived from 2D X-ray angiography images based on the CFD method, assuming rigid arterial walls and steady-state blood flow. They found that an increase in the degree of stenosis led into a reduction of flow and pressure and concluded that occlusion created an eddy in the flow pattern of blood. Morris et al. [5] evaluated hemodynamic parameters such as pressure and FFR in different degrees of stenosis on X-ray angiography and found a drop in pressure and FFR in the post-stenosis portion of the right coronary artery in the hyperemic state. In the present study, blood was assumed to be nonNewtonian and steady-state flow and arterial walls were assumed to be rigid. Young et al.
[6] conducted an angiography-derived numerical simulation of the blood flow in the LAD with the assumption that blood was a Newtonian fluid in arteries with rigid walls under a steady state. They found a strong correlation between computationally simulated FFR and pressure-wire-based FFR in the hyperemic state.

The aim of the current study was to present a new approach on the basis of 3D coronary angiography and the Thrombolysis in Myocardial Infarction (TIMI) frame count for fast computation of FFR in patients with coronary artery disease. The accuracy of computationally simulated FFR was evaluated in patients with intermediate coronary stenosis using pressure-wire-based FFR as the reference standard.

\section{Material and Methods}

\section{Construction of Geometry}

In this simulation study, two projections of patient-specific X-ray angiography images were obtained to construct computational geometry for the numerical simulation of the blood flow. From each angiographic projection, a single end-diastolic frame was manually selected using Visionlite software. For the conversion of the pixel size to the millimeter, a scaling factor was determined. The $3 \mathrm{D}$ centerline of the curvature of the coronary arteries was derived from the curve of the projection of the 2 views using Rhinoceros, version 5.0. The lumen borders were manually detected in each angiographic projection, and the lumen centerline was calculated in each projection (Figure 1A). Then, the 2D circular crosssection perpendicular to the centerline of the artery was extracted. The resulting 3D lumen volume generated meshes, which were thereafter exported to the CFD solver (Figure 1B).

\section{Governing Equations}

In this study, numerical simulation was conducted under a steady condition. Arterial walls 


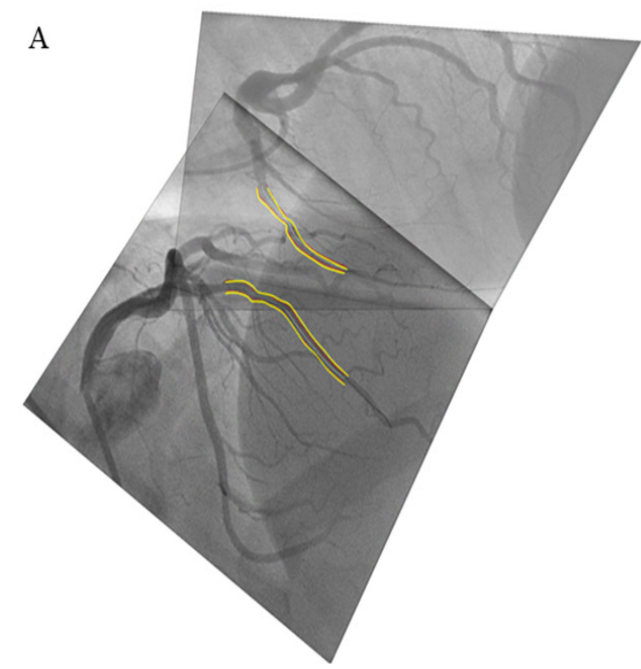

B

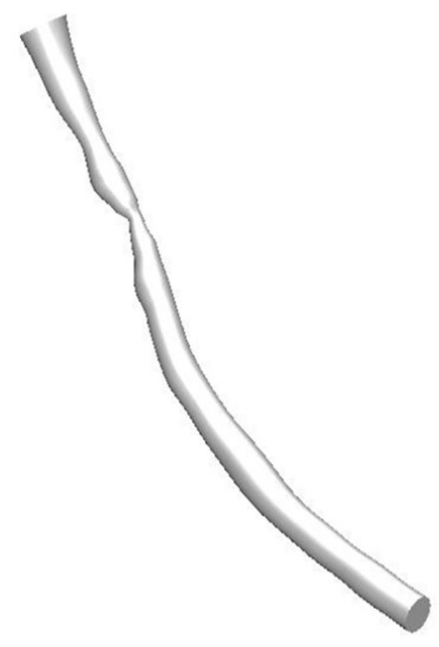

Figure 1: Coronary artery geometry from X-ray angiography images: $(A)$ two projections (B) 3D model

were defined as rigid and no-slip. Blood was assumed to be an incompressible, laminar, and non-Newtonian fluid. The motion of the blood flow was assumed to be governed by continuity equation 1 and momentum equations 2 [7].

$$
\begin{aligned}
& u_{i, j}=0 \\
& \rho\left(\frac{\partial u_{i}}{\partial t}+u_{j} u_{i, j}\right)=-p_{i}+\tau_{i j, j}
\end{aligned}
$$

Where $u, \rho$, and $\tau$ are blood velocity, blood density and the stress tensor, which are defined by equation 3 :

$$
\tau_{i j}=\mu_{\mathrm{eff}} \dot{\gamma}_{i j}
$$

Where $\mu_{\text {eff }}$ and $\gamma$ denote the viscosity of blood and the shear rate, respectively. In this work, the relation between $\mu_{\text {eff }}$ and $\gamma^{\circ}$ is described by equation 4:

$$
\mu_{\text {eff }}=\mu_{\text {eff }}+\left(\mu_{0}-\mu_{\infty}\right)\left[1+(\lambda \dot{\gamma})^{2}\right]^{\frac{(n-1)}{2}}
$$

Where $\mu_{0}=0.056$ Pa.s, $\mu_{\infty}=0.00354$ Pa.s $\lambda=3.313 \mathrm{~s}$, and $n=0.3568$ and the shear rate $\gamma$ is defined by equation 5 :

$$
\dot{\gamma}=\sqrt{2 \operatorname{tr}\left[\frac{1}{2}\left(\nabla u+(\nabla u)^{T}\right)\right]^{2}}
$$

\section{Boundary Condition}

The boundary condition of the inlet The motive force of the blood flow is the pressure gradient in the aorta and the coronary artery. The outflow of the left ventricle is not always uniform because the aortic valve creates flow disturbance. Although the exact effects of the aortic valve cannot be thoroughly simulated, the uniform inflow at the inlet of the coronary artery can be ignored using pressure inlet boundary condition, which is similar to previous studies. Thus, the pressure inlet boundary condition in the present study is realistic [8]. The mean arterial pressure (MAP) defined as the average pressure over the cardiac cycle is responsible for driving blood into the distal vessels and ultimately in the tissues [9]. The MAP is estimated from equation 6:

$\mathrm{MAP}=\mathrm{DBP}+\left(\frac{1}{3}+(\mathrm{HR} \cdot 0.0012)\right) \cdot(\mathrm{SBP}-\mathrm{DBP})$

Where HR, SBP and DBP are the heart rate, the systolic blood pressure and the diastolic blood pressure, respectively. The contrast transport time in the reconstructed branch of the coronary artery was calculated on rest projections using the TIMI frame count [10]. The measured FFR values were considered blind. 
Figures 2 F1 to F9 show consecutive image frames in the resting state. The mean volumetric flow rate (VFR) in rest state was derived from the lumen volume of the reconstructed coronary tree divided by the contrast transport time.

The boundary condition of the coronary outlet

Electrical circuit analogy was used to model the downstream arteries derived from the 3D model. This analogy created a relationship between pressure and the flow rate. Lumped (0D) boundary conditions were specified at the outlet of each stenosis artery. The resting total coronary blood flow was calculated by the TIMI frame count of the X-ray angiography images [11]. Hence, the branch resistance of each patient (R) was computed from the MAP and the mean flow $(\mathrm{Q})$ :

$$
\mathrm{R}=\frac{\mathrm{MAP}}{\mathrm{Q}}
$$

In assigning boundary conditions, the final step was to simulate maximum hyperemia by modeling the effect of adenosine on reducing the peripheral resistance of the downstream coronary arteries extracted from the X-ray angiography images. Wilson et al. [12] showed that for arteries in which the resistance would be expected to be small both at rest and during hyperemia, total coronary resistance at maximum hyperemia fell to 0.21 of the resting value with an intravenous administration of $140 \mathrm{mg} / \mathrm{kg} / \mathrm{min}$ of adenosine. Hyperemic resistance is given by:

$\mathrm{R}_{\text {Hyperemia, New }}=(1-\alpha) \cdot \mathrm{R}_{\text {Hypermia, Old }}+\alpha\left(\mathrm{K} \cdot \frac{\mathrm{MAP}}{\mathrm{VFR}}\right)(8$

Here, $\alpha$ is the under-relaxation factor, $\mathrm{R}_{\text {Hypermia, Old }}$ represents resistance in the last iteration, $\mathrm{R}_{\text {Hypermia, New }}$ represents this value in the next iteration and VFR stands for the volumetric flow rate.

The user-defined function was compiled to supply the resistance boundary conditions at the outlets. The downstream vasculature of
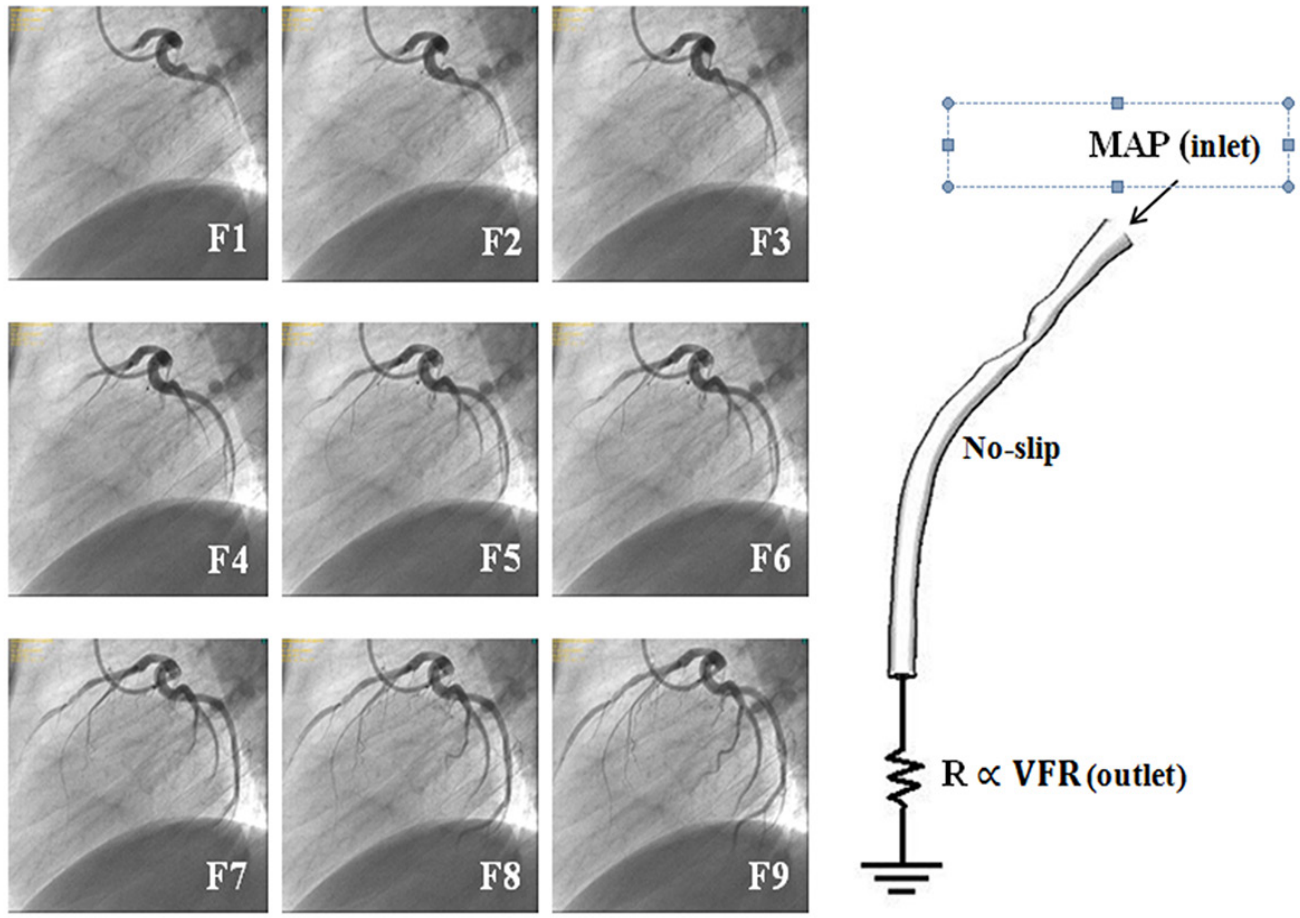

Figure 2: Boundary condition based on the Thrombolysis in Myocardial Infarction (TIMI) frame count of the angiographic images 
the primary branches was coupled to the LAD model, and the static pressure was solved iteratively at each outlet. Smooth convergence was ensured by attenuating the pressure gradient calculated at the outlet several times to couple conditions of iterative under-relaxation-based resistance boundary with those outlets. In other words, an implicit algorithm was applied to the outlet with an under-relaxation factor given as:

$$
\mathrm{P}_{\mathrm{k}+1}=(1-\alpha) \cdot \mathrm{P}_{\mathrm{k}}+\alpha\left(\mathrm{R}_{\text {Hypermia }} \cdot \mathrm{Q}_{\mathrm{k}}-\mathrm{P}_{\mathrm{k}}\right)
$$

The numerical solution to equations 8 and 9 was carried out with an explicit Euler scheme. Subsequently, the equations were applied using FLUENT software in the form of user-defined function.

\section{Computations}

The numerical calculations were carried out using the finite volume method in ANSYS FLUENT software. Temporal and spatial discretization were performed with a second-order backward Euler scheme and second-order central differencing, respectively. The pressure was solved through the pressure-veloci-
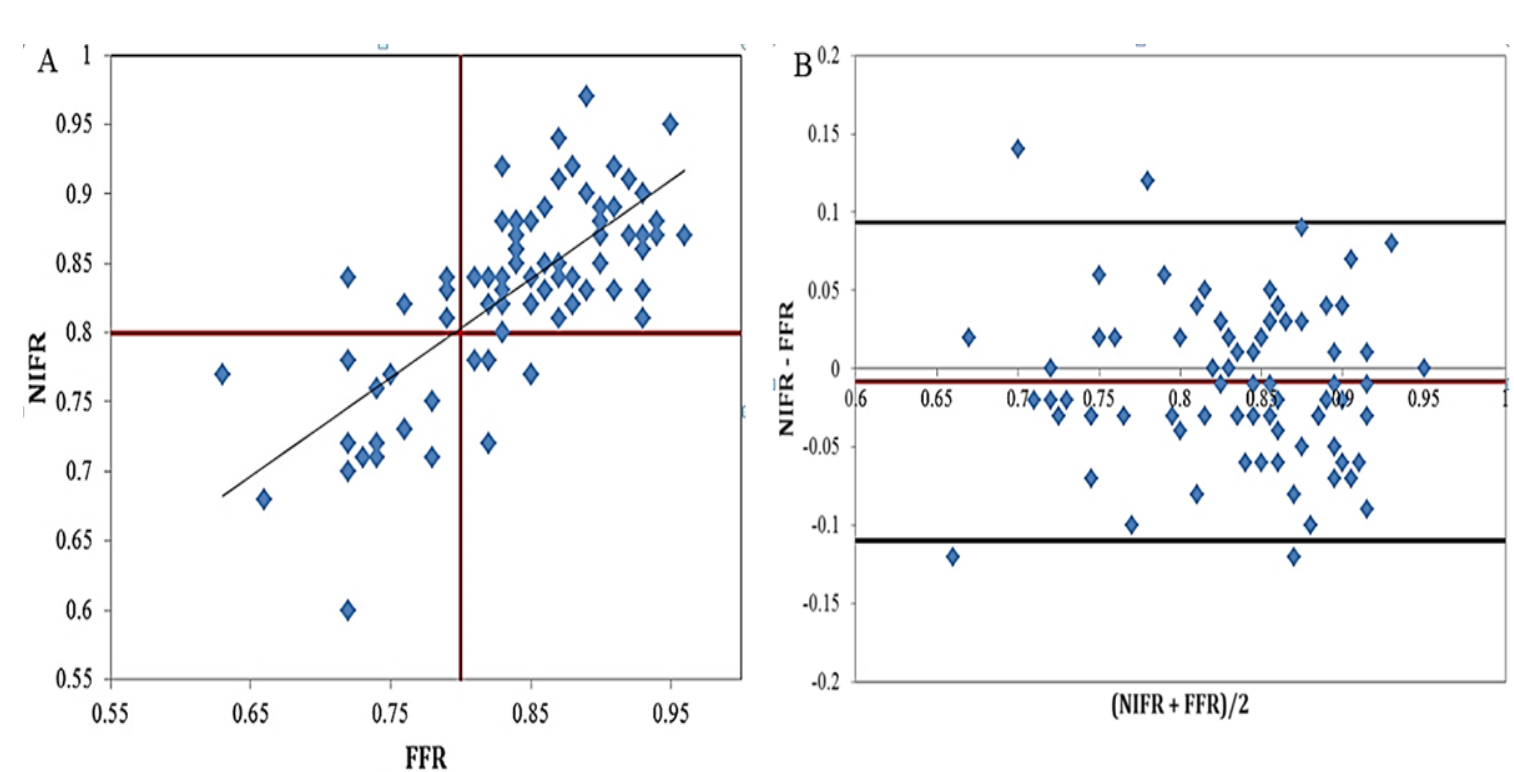

ty coupling method known as the "SIMPLE algorithm". The residual of the solution was kept at 0.00001 , and the simulations were progressed under a steady-state condition. The computational mesh utilized in the primary simulations consisted of nearly 300,000 triangular elements for diseased geometry. The triangular computational meshes were constructed in ANSYS ICEM CFD 16.0 (ANSYS Workbench). The simulations were performed by a personal computer with a $2.5 \mathrm{GHz}$ Core i5 Duo processor and 4 GB of RAM.

\section{Results}

Statistical analysis: correlation between NIFR with pressure-wirebased FFR

In the current study, FFR was calculated based on CFD derived from X-ray images in patients with intermediate stenosis in the LAD and the right coronary artery. The statistical analyses were performed using SPSS (version 20.0.0). The Pearson correlation was applied to evaluate the correlation between NiFR and pressure-wire-based FFR. There were several main findings. Figure $3 \mathrm{~A}$ shows the correlation

Figure 3: A) Correlation between pressure-wire-based fractional flow reserve (FFR) and NonInvasive Flow Ratio (NiFR); B) Bland-Altman plot for the agreement of pressure-wire-based FFR and NiFR. 
between NiFR and pressure-wire-based FFR. The NiFR ( $\mathrm{r}=0.738, \mathrm{P}<0.001)$ exhibited a strong correlation with pressure-wire-based FFR. The result indicated that FFR was higher than 0.8 in the arteries with non-significant stenosis and lower than 0.8 in the arteries with significant stenosis. The NiFR (mean difference $=-0.00821$, standard deviation $=0.0931$ ) (Figure 3B) demonstrated a slight difference but non-significant as compared with FFR in assessing the functional significance of stenosis on a per-vessel basis.

\section{Discussion}

Patients of coronary artery disease (CAD) may have one of the following on diagnostic coronary angiography: normal or mildly diseased coronary arteries $(0 \%$ to $30 \%$ diameter stenosis), stenosis of intermediate severity $(30 \%-60 \%)$ and significant stenosis (60\%-100\%). The visual assessment based on the geometry of stenosis can be sufficient for diagnostic significant and non-significant in the cases of normal and severe coronary, respectively. But, in many instances, visual and quantitative assessment alone may be insufficient to establish stenosis with a moderate degree. Therefore, FFR should be measured to identify the hemodynamic parameters of lesions and the impact of stenosis on the parameters, such as the impact of the lesion on the pressure and flow drop. We have developed a model that indicates the feasibility of using image base analysis and CFD techniques to predict physiological state in coronary disease. In this study, pressure and flow can be calculated by a CFD solver, with generic boundary condition. There are several advantages offered using physiological measures, which were derived from our CFD method. This method only requires coronary geometry. There is no need for the hyperemia flow, additional procedure time and pressure wire. The current study has some limitations that the most important onesare relatively small population and retrospective design. Another drawback is that only patients with lesions in the LAD were recruited. Therefore, more data are required to expand this application to other coronary vessels. Moreover, our model was constructed without taking myocardial movement into account. Other studies have shown that myocardial motion exerts minor effects on the flow distribution as compared with the effects of boundary conditions applied [13]. Moreover, arterial walls were assumed to be rigid in our model. According to other studies, however, comparisons of the computational results between fluid-structure interaction and rigid-wall models have shown that instantaneous (wall shear stress) distributions are affected by diameter variations in the arterial wall. The effects of wall compliance on timeaveraged wall shear stress (TAWSS) and the oscillatory shear index (OSI) are insignificant (4.5 and $2.7 \%$ difference in maximum TAWSS and OSI, respectively) [14, 15].

\section{Conclusion}

In the present study, FFR calculated based on CFD was utilized to assess the impact of stenosis on blood pressure and flow. Although regarded as the gold standard method, FFR is used in less than $10 \%$ of PCI procedures because of its costs and invasive nature. According to CFD, we calculated NiFR in 85 patients. The results of the diagnostic computational procedure demonstrated that FFR was higher than 0.8 for patients with a normal condition and lower than 0.75 for patients with significant stenosis. This method was used to increase the utility of FFR assessment for patients with intermediate and borderline coronary stenosis. The computational simulation of FFR and hemodynamic parameters such as pressure drop is a safe, efficient, and costeffective method to evaluate the severity of coronary stenosis.

\section{Acknowledgment}

The authors sincerely thank the staff of the cardiac catheterization laboratory at Imam 
Khomeini Hospital, Tehran, Iran.

\section{Authors' Contribution}

Mohamamd Sadeghian conceived the idea study concept, and supervised all the steps of the study. Vahid Mohammadi wrote the proposal, and collected the data collection. Akbar Shafiee performed the data analysis, drafted the manuscript, and did the revisions. Hamidreza babakhani did the data coollection and image processing, and programmed the software.

All the authors read and approved the initial and final draft of this manuscript.

\section{Ethical Approval}

The Ethics Committee of Tehran University of Medical Sciences approved the protocol of the study (IR.TUMS.IKHC.REC.1397.005).

\section{Funding}

This study was supported by Tehran University of Medical Sciences, Tehran, Iran (Grant for proposal No. 1160).

\section{Conflict of Interest}

None

\section{References}

1. Pijls NH, Fearon WF, Tonino PA, Siebert U, Ikeno F, Bornschein $B$, et al. Fractional flow reserve versus angiography for guiding percutaneous coronary intervention in patients with multivessel coronary artery disease: 2-year follow-up of the FAME (Fractional Flow Reserve Versus Angiography for Multivessel Evaluation) study. J Am Coll Cardiol. 2010;56:177-84. doi: 10.1016/j.jacc.2010.04.012. PubMed PMID: 20537493.

2. Min JK, Leipsic J, Pencina MJ, Berman DS, Koo BK, Van Mieghem C, et al. Diagnostic accuracy of fractional flow reserve from anatomic CT angiography. JAMA. 2012;308:1237-45. doi: 10.1001/2012. jama.11274. PubMed PMID: 22922562. PubMed PMCID: PMC4281479.

3. Dehmer GJ, Weaver D, Roe MT, Milford-Beland S, Fitzgerald $S$, Hermann A, et al. A contemporary view of diagnostic cardiac catheterization and percutaneous coronary intervention in the United States: a report from the CathPCl Registry of the National Cardiovascular Data Registry, 2010 through June 2011. J Am Coll Cardiol. 2012;60:2017-31. doi: 10.1016/j.jacc.2012.08.966. PubMed PMID:
23083784

4. Tu S, Pyxaras SA, Li Y, Barbato E, Reiber JH, Wijns $W$. In vivo flow simulation at coronary bifurcation reconstructed by fusion of 3-dimensional $X$-ray angiography and optical coherence tomography. Circ Cardiovasc Interv. 2013;6:e15-17. doi: 10.1161/CIRCINTERVENTIONS.112.000051. PubMed PMID: 23591422.

5. Morris PD, Ryan D, Morton AC, Lycett R, Lawford PV, Hose DR, et al. Virtual fractional flow reserve from coronary angiography: modeling the significance of coronary lesions: results from the VIRTU-1 (VIRTUal Fractional Flow Reserve From Coronary Angiography) study. JACC Cardiovasc Interv. 2013;6:149-57. doi: 10.1016/j. jcin.2012.08.024. PubMed PMID: 23428006.

6. Yong AS, Ng AC, Brieger D, Lowe HC, Ng MK, Kritharides L. Three-dimensional and two-dimensional quantitative coronary angiography, and their prediction of reduced fractional flow reserve. Eur Heart J. 2011;32:345-53. doi: 10.1093/eurheartj/ ehq259. PubMed PMID: 20705695.

7. Carneiro F, Ribeiro VG, Teixeira J, Teixeira S. Numerical study of blood fluid rheology in the abdominal aorta. Design \& Nature IV: Comparing Design in Nature with Science and Engineering. 2008;4:169. doi: 10.2495/dn080181.

8. Vasava P, Jalali P, Dabagh M, Kolari PJ. Finite element modelling of pulsatile blood flow in idealized model of human aortic arch: study of hypotension and hypertension. Comput Math Methods Med. 2012;2012:1-14. doi: 10.1155/2012/861837. PubMed PMID: 22400055. PubMed PMCID: PMC3287084.

9. Itu L, Sharma P, Ralovich K, Mihalef V, Ionasec $R$, Everett $A$, et al. Non-invasive hemodynamic assessment of aortic coarctation: validation with in vivo measurements. Ann Biomed Eng. 2013;41:669-81. doi: 10.1007/s10439-012-07150. PubMed PMID: 23232558.

10. Gibson CM, Cannon CP, Daley WL, et al. TIMI frame count: a quantitative method of assessing coronary artery flow. Circulation. 1996;93(5):87988. doi: 10.1161/01.cir.93.5.879. PubMed PMID: 8598078.

11. Kim HJ. Three-dimensional finite element modeling of blood flow in the coronary arteries. United States, California Stanford: University; 2009.

12. Wilson RF, Wyche K, Christensen BV, Zimmer S, Laxson DD. Effects of adenosine on human coronary arterial circulation. Circulation. 1990;82:1595606. doi: 10.1161/01.cir.82.5.1595. PubMed PMID: 2225364. 
13. Theodorakakos A, Gavaises M, Andriotis A, Zifan A, Liatsis P, Pantos I, et al. Simulation of cardiac motion on non-Newtonian, pulsating flow development in the human left anterior descending coronary artery. Phys Med Biol. 2008;53:487592. doi: 10.1088/0031-9155/53/18/002. PubMed PMID: 18711245.

14. Torii R, Wood NB, Hadjiloizou N, Dowsey AW, Wright AR, Hughes AD, et al. Fluid-structure interaction analysis of a patient-specific right coronary artery with physiological velocity and pres- sure waveforms. Communications in Numerical Methods in Engineering. 2009;25:565-80. doi: 10.1002/cnm.1231.

15. Siogkas PK, Papafaklis MI, Sakellarios AI, Stefanou KA, Bourantas CV, Athanasiou LS, et al. Patient-specific simulation of coronary artery pressure measurements: an in vivo three-dimensional validation study in humans. Biomed Res Int. 2015;2015:1-11. doi: 10.1155/2015/628416. PubMed PMID: 25815328. PubMed PMCID: PMC4359837. 